\title{
Sofia Navalho
}

sofianavalho28@gmail.com

Casa-Museu Soledade Malvar: edifício, coleção e inventário. 
Resumo

Este texto tem como objetivo descrever o processo de desenvolvimento do Relatório de Estágio intitulado "Casa-Museu Soledade Malvar - Edifício, Coleção e Inventário", elaborado no âmbito do Mestrado de Museologia da FLUP. O Relatório centra-se no estudo da história da Casa-Museu Soledade Malvar e de uma pintura pertencente ao seu acervo. Este foca-se, igualmente, no processo de leitura, análise e revisão das mais recentes fichas de inventário da casa-museu.

O contributo inicia-se com uma descrição do tema da investigação e com uma apresentação da casamuseu e da história da sua criação. Segue-se a descrição pormenorizada do processo de desenvolvimento do Relatório, mencionando todas as etapas que levaram ao resultado final. Por fim, são apresentadas as considerações finais, refletindo sobre as dificuldades que surgiram e sobre os contributos que resultaram da investigação desenvolvida.

Palavras-chave: Estudo de coleção; Inventário; CasaMuseu; Museologia

\section{Nota biográfica}

Sofia Navalho é Licenciada em História da Arte e Mestre em Museologia, pela Faculdade de Letras da Universidade do Porto. Durante o seu 2.ㅇ ciclo de estudos, no Mestrado em Museologia, realizou um Estágio na Casa-Museu Soledade Malvar, instituição museológica pertencente à Rede de Museus de Vila Nova de Famalicão. Desenvolveu ainda algumas atividades com esta mesma rede de museus, tais como a criação e gravação de uma visita guiada intitulada "Um riacho - O naturalismo de Júlio Ramos" e a publicação de um artigo, que integrará o Boletim Cultural de Vila Nova de Famalicão, de 2021.

\section{Abstract}

This text has the purpose of demonstrating the process of the development of the Internship Report intitled "Casa-Museu Soledade Malvar - Edifício, Coleção e Inventário", created within the scope of the master's degree in Museology at FLUP. This Report focuses on the study of the history of the Casa-Museu Soledade Malvar and a painting that belongs to its collection. It focuses, as well, on the process of reading, analysing and reviewing the most recent inventory sheets from this house museum. The contribute starts with a description of the research's theme and a presentation of the CasaMuseu Soledade Malvar and the history of its creation. After that, there is a description of the report's development process, mentioning all the steps that led to its results. Lastly, final considerations are presented, reflecting on the difficulties and on the contributions that resulted from the developed research.

Keywords: Collection studies; Inventory; House Museum; Museology

\section{Biographical note}

Sofia Navalho has a degree in Art History and a master's degree in Museology, from the Faculty of Arts and Humanities of the University of Porto. During her $2^{\text {nd }}$ cycle of studies, the Masters in Museology, she held an internship in Casa-Museu Soledade Malvar, a museological institution belonging to the Vila Nova de Famalicão Museum Network.

She also developed some activities with the same museum network, such as the creation and recording of a guided tour entitled "A stream - The naturalism of Júlio Ramos" and the publication of an article, which will be included in the Cultural Bulletin of Vila Nova de Famalicão, of 2021. 


\section{Introdução}

Este contributo tem como propósito partilhar o essencial do processo que levou à elaboração do Relatório de Estágio, desenvolvido no âmbito da conclusão do Mestrado em Museologia (MMUS) da Faculdade de Letras da Universidade do Porto (FLUP), sob a orientação de Alice Semedo e Mariana Jacob (Navalho, 2020). O Relatório, apresentado em finais de 2020, resulta de um estágio curricular, de 400 horas de duração, realizado na Casa-Museu Soledade Malvar. Os temas abordados nesse documento focam-se no estudo da história da casa-museu e das suas transformações, assim como no de uma pintura pertencente ao seu acervo, da autoria do pintor naturalista Júlio Ramos, centrando-se, igualmente, na leitura e revisão crítica das mais recentes fichas de inventário de pintura da instituição museológica.

Este texto inicia-se com uma breve apresentação da Casa-Museu Soledade Malvar e da história da sua criação, com o objetivo de a dar a conhecer aos leitores. Nela, inclui-se também uma pequena biografia da antiquária e colecionadora, que dá nome à instituição e que é a responsável pela sua existência. De seguida, são indicados os motivos que levaram à seleção da instituição como local de estágio, os objetivos de trabalho e a descrição das várias fases de desenvolvimento, apresentando os vários conceitos analisados e o processo de seleção do modelo de estudo de coleções que serviu de base ao estudo da pintura "Um riacho" (Milheiroz). Posteriormente, são descritas as quatro fases de investigação sobre a pintura mencionada, referindo as informações recolhidas em cada uma e as dificuldades que foram surgindo ao longo desse processo, refletindo sobre as alternativas adotadas para as ultrapassar.

\section{A Casa-Museu Soledade Malvar}

A Casa-Museu Soledade Malvar (CMSM) surge de um interesse, por parte da antiquária e colecionadora, Maria da Soledade Ramos Malvar Osório, de criar um 
museu, com o intuito de doar à comunidade famalicense a sua coleção (Rede de Museus de Vila Nova de Famalicão, 2018).

Figura conhecida em Vila Nova de Famalicão, de onde é natural, Maria da Soledade desenvolveu um particular interesse pela arte, desde a sua infância. O gosto pelas antiguidades, que foi desenvolvendo ao longo da vida, culmina na abertura de um Bricà-Brac, frequentado por importantes figuras nacionais (Rede de Museus de Vila Nova de Famalicão, 2002). Esta vontade de criar uma instituição museológica é concretizada em 1998, a partir da elaboração de uma escritura, onde foi acordada a doação, ao Município de Vila Nova de Famalicão, do prédio urbano pertencente a Maria da Soledade e dos 414 objetos pertencentes à sua coleção. Estes foram seccionados em 3 categorias, designadas por “Mobiliário, Porcelanas e Esculturas”, "Ourivesaria” e “Pintura” (Câmara Municipal de Vila Nova de Famalicão, 1998).

A 29 de setembro de 2002, a casa-museu é inaugurada, após um período de obras de adaptação do edifício à função museológica, realizado com o propósito de respeitar o traçado original do edifício, de forma que o ambiente doméstico vivenciado pela colecionadora se mantivesse (Rede de Museus de Vila Nova de Famalicão, 2018). As obras tiveram uma duração de cerca de dois anos, tendo sido executadas pela Firma Andrade \& Almeida - Construções Lda. (Câmara Municipal de Vila Nova de Famalicão, 2000), cujo projeto foi selecionado a partir da abertura de um concurso público. Durante esta fase de construção e remodelação, os objetos doados por Maria da Soledade foram transferidos para um apartamento seu, à exceção das peças de joalharia (Câmara Municipal de Vila Nova de Famalicão, s.d.). Algumas das alterações executadas respeitam a transformação do anterior Bric-à-Brac numa sala de exposições temporárias, ou a demolição do quarto de vestir. Ainda durante este período, o anterior quarto de hóspedes foi convertido na sala onde atualmente são expostos os objetos de joalharia (Câmara Municipal de Vila Nova de Famalicão, 2000).

A CMSM pertence à Rede de Museus de Vila Nova de Famalicão (RMVNF), criada em novembro de 2012, na procura de tornar o património cultural famalicense um 
potenciador de visitação turística (Câmara Municipal de Vila Nova de Famalicão, 2012). A RMVNF demonstra uma preocupação com a proteção da herança patrimonial de Vila Nova de Famalicão, com a comunidade e o com desenvolvimento da mesma, a partir da utilização de um sistema pedagógico e educativo. Acresce a estas preocupações a vontade de promover a identidade do concelho e da sua comunidade (Câmara Municipal de Vila Nova de Famalicão, 2012). É composta por 12 unidades museológicas, com caráter de coleção visitável, ou de museu.

A CMSM localiza-se no número 104 da Avenida 25 de abril (Fig. 1) e é composta por uma grande variedade de objetos, anteriormente pertencentes à colecionadora e antiquária Maria da Soledade Malvar (Rede de Museus de Vila Nova de Famalicão, 2018).

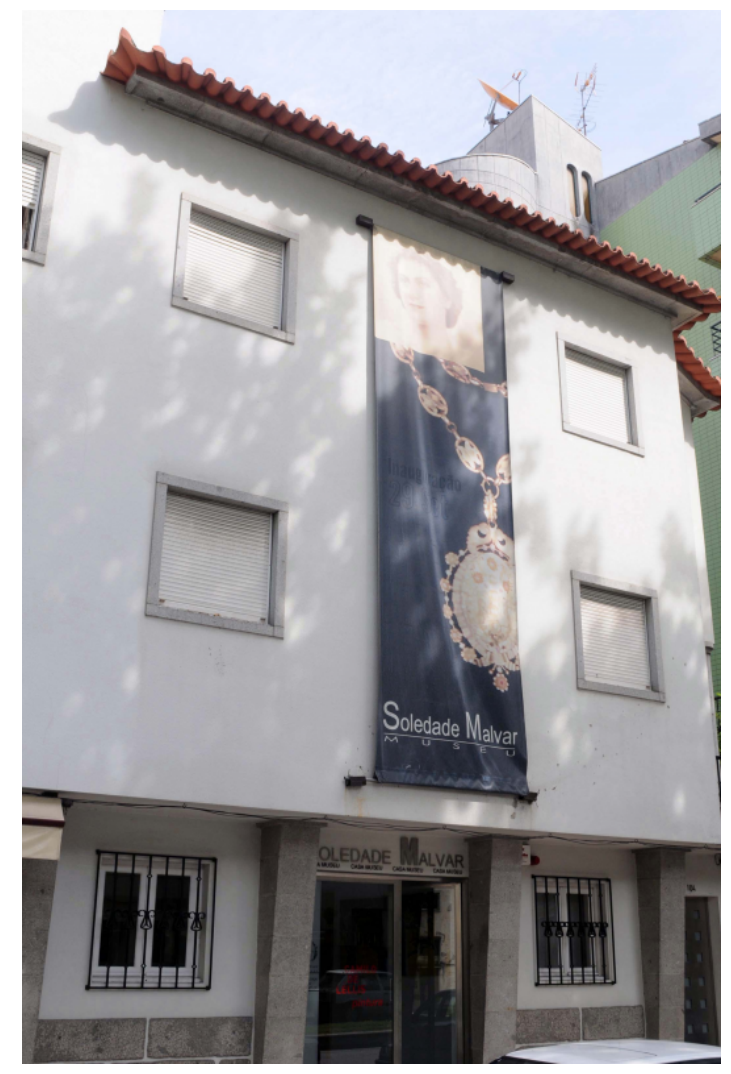

Fig. 1 - Fachada da Casa-Museu Soledade Malvar (Câmara Municipal de Vila Nova de Famalicão, s.d.). 
Entre os 553 objetos em causa, encontram-se algumas esculturas, das quais se destaca a imagem de Santo António, exposta em local de destaque, no piso térreo do edifício. A coleção é também composta por objetos das categorias Numismática e Têxteis (incluindo vestuário de criança e tapeçarias). O mobiliário apresenta-se em grande número e abrange uma diversidade de objetos, como cadeiras e diferentes tipos de cómodas e mesas, de maior ou menor dimensão. Os objetos de cerâmica estão expostos um pouco por todo o edifício, mas concentram-se, sobretudo, num armário de grandes dimensões, situado no $1 \cong$ piso do museu. As peças de ourivesaria localizam-se no $2 \cong$ piso, numa sala própria, que apresenta condições de segurança específicas, devido à natureza deste tipo de objetos. A restante coleção inclui algumas fotografias (que retratam membros da família de Maria da Soledade, ou a própria colecionadora), gravuras e algumas peças que se inserem nas categorias de Metais e de Vidros.

Todos estes objetos se encontram inventariados - tendo em conta as normas de preenchimento de dados incluídas no programa In Patrimonium.Net - e foram adquiridos em diversos antiquários (Câmara Municipal de Vila Nova de Famalicão, 2002) ou em leilões. A grande maioria encontra-se em exposição permanente, estando disposta pelo rés-do-chão e, sobretudo, pelos 10 e 20 pisos do edifício. À exceção da coleção de ourivesaria, todos os objetos, das mais variadas categorias, convivem nas mesmas salas de exposição desta casa-museu.

A coleção de pintura é composta por 21 objetos, de diferentes formatos e dimensões, cuja maioria data do século XIX e XX. Muitas destas peças integram o acervo da casamuseu desde 1997, embora a maior parte tenha sido doada apenas em 2003. À exceção de uma pintura a aguarela sobre papel, todas as restantes foram executadas a óleo, sendo, sobretudo, a óleo sobre tela (14, no total). As restantes são a óleo sobre pergaminho, cobre ou madeira. As temáticas abordadas são a religiosa, que se apresenta em maior número, seguida da natureza-morta, da paisagem e do retrato. A maioria encontra-se em exposição permanente e distribui-se por toda a casa-museu, embora se concentre, maioritariamente, na sala de jantar, localizada no 10 piso do 
edifício. Em reserva, encontram-se apenas 6 pinturas, envoltas em espuma de polietileno. Algumas estão colocadas numa estante de metal e as restantes estão encostadas a uma parede e pousadas sobre placas de cartão, posicionadas no chão.

A CMSM pretende transmitir aos visitantes os conhecimentos sobre Maria da Soledade Malvar e divulgar o seu legado (Rede de Museus de Vila Nova de Famalicão, 2019). Do mesmo modo, a casa-museu procura ser um "lugar de encontro entre gerações", onde se tenciona alcançar o "desenvolvimento pleno de cada pessoa na sua relação com a comunidade local” (Rede de Museus de Vila Nova de Famalicão, 2019, p. 49).

\section{Metodologia}

O processo de desenvolvimento do Relatório de Estágio teve início no momento em que foi selecionado o local de Estágio e em que foram ponderados os temas a abordar. A escolha da Casa-Museu Soledade Malvar derivou de uma visita ao local e de uma conversa com uma das responsáveis pela coordenação da RMVNF. Durante o primeiro contacto com os objetos da coleção do museu, aqueles que despertaram maior interesse foram as pinturas, que não se encontravam estudadas. Deste modo, optouse por realizar um estudo sobre as mesmas e, ainda, uma investigação relativa à história do edifício.

Uma das fases iniciais desta investigação prendeu-se com o delinear dos objetivos. Estes basearam-se na tentativa de contribuir para a recolha de informações sobre a pintura estudada e sobre a história da Casa-Museu Soledade Malvar, procurando desenvolver uma investigação que resultasse na introdução de novos dados nas fichas de inventário do museu.

De forma a organizar as várias tarefas de investigação, desenvolveu-se um cronograma, que acabou por não ser cumprido da forma prevista. Registaram-se alguns atrasos, devido a obstáculos decorrentes da pandemia de COVID-19. 
A fase que se seguiu focou-se na elaboração de uma reflexão teórica relativa ao conceito de coleção e a várias questões relacionadas com o tema. A leitura e interpretação de vários textos, especialmente de Pearce (2003a; 2003b; 2003c) e Belk (2003), permitiu produzir uma reflexão sobre os vários modos de colecionar, as motivações subjacentes à prática de colecionar e o significado e importância que os objetos possuem.

De seguida, foram estudados textos que apresentam diferentes abordagens ao tema da cultura material. O objetivo foi demonstrar a forma como, dentro do mesmo tema, se podem registar perspetivas muito distintas. Pela sua relevância, destacam-se os de Appadurai (1986) e de Kopytoff (1986).

Posteriormente e tendo em consideração que uma parte significativa da investigação se centrava na história da Casa-Museu Soledade Malvar, tornou-se fundamental efetuar uma reflexão sobre o conceito de casa-museu e explicar por que razão é que esta instituição museológica se enquadra nesse contexto. Para tal, revestiram-se de grande importância as publicações de Ponte (2007; 2019) e de Günhan (2011).

Foram também considerados alguns modelos de estudo de coleções, como os de Batchelor (2003), Elliot (2003), Pearce (2003) e Prown (2003). O modelo de Pearce foi o adotado para a realização do estudo de uma pintura, por se considerar ser o que melhor se adequava ao tipo de objeto selecionado e que oferecia uma análise mais completa do mesmo.

O processo de seleção de uma das pinturas não estudadas resultou da consulta das informações existentes nas mais recentes fichas de inventário da CMSM. Após observar a escassez de dados referentes à grande maioria dos objetos, optou-se por efetuar um estudo sobre a obra "Um riacho" (Milheiroz), de Júlio Ramos (Fig. 2). 


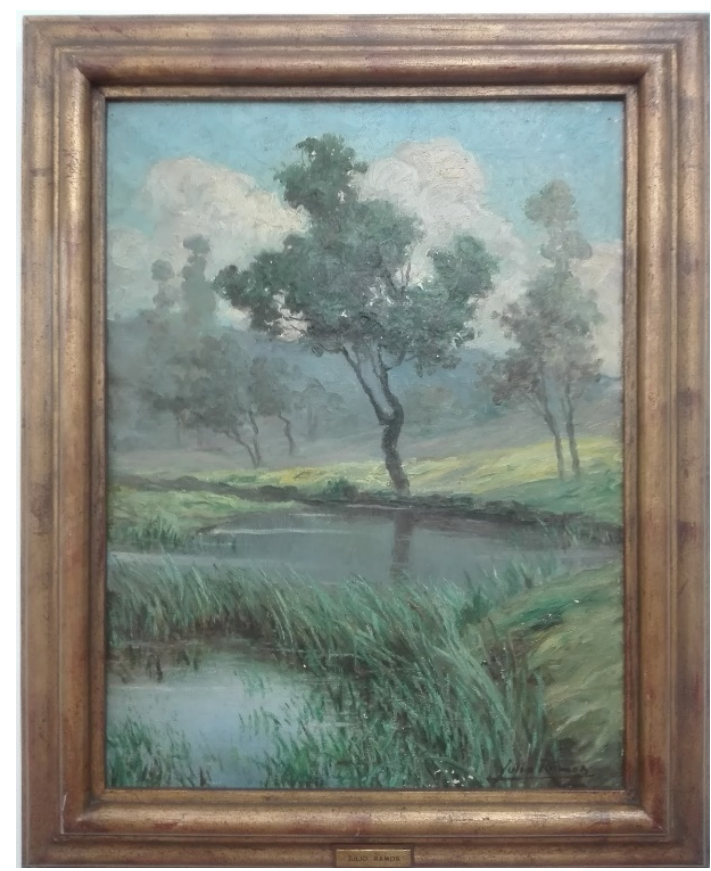

Fig. 2 - Pintura "Um riacho" (Milheiroz), de Júlio Ramos. (C Sofia Navalho, 2019.

Tratando-se de uma pintura assinada de um autor reconhecido, depreendeu-se que existiriam informações suficientes para se proceder ao seu estudo. O modelo de Pearce foi adaptado às características do objeto e às informações que foram sendo recolhidas, mantendo-se a sua organização em 4 fases distintas: o "material", a "história" - seccionada em dois momentos -, o "contexto" e o "significado". A primeira fase focou-se na observação atenta e na descrição detalhada do objeto, a partir do seu exame. De seguida, foram consultadas algumas obras de artistas naturalistas, como Jean-Baptiste Corot e Charles-François Daubigny, de modo a estabelecer uma comparação entre a pintura em estudo e outras que se inserem no mesmo movimento artístico. De seguida, procurou-se consultar a obra artística de Júlio Ramos, com o objetivo de compreender as suas principais características e de estabelecer uma comparação entre as várias obras e a pintura "Um riacho" (Milheiroz). Devido à 
situação pandémica e ao encerramento de instituições museológicas, as pinturas existentes nos acervos dos museus não puderam ser consultadas presencialmente ${ }^{1}$.

Para esta comparação, utilizaram-se as pinturas retratadas no catálogo da exposição de homenagem a Júlio Ramos (Salão Silva Porto, 1943) e algumas apresentadas no site da Leiloeira S. Domingos (s.d.).

A fase que se refere à "história" foi seccionada em dois momentos distintos. $\mathrm{O}$ primeiro abordou a história geral do objeto, procurando aprofundar os conhecimentos sobre o seu autor e recolher informações sobre os proprietários anteriores. Para este fim, foram contactados o Ateneu Comercial do Porto e alguns antiquários e leilões das regiões de Vila Nova de Famalicão, Braga e Porto. Porém, as respostas foram escassas e nenhuma informação pôde ser recolhida a partir deste método. Recolheu-se, também, informação sobre a biografia de Júlio Ramos, referente ao seu percurso académico (Universidade Digital/Gestão de Informação, 2009) e à sua carreira artística (Pamplona, 1991).

O segundo momento focou-se na história da pintura, desde o momento em que passou a fazer parte do acervo da CMSM. A partir de testemunhos orais e escritos de antigos funcionários da instituição, procurou saber-se o histórico das movimentações e transformações que o objeto foi tendo, questionando os intervenientes sobre os locais onde a pintura foi exposta ou sobre a possibilidade de ter sofrido algum restauro.

A fase do "contexto" foi marcada pelo estudo do contexto histórico e cultural da corrente artística em que se enquadra a pintura. A partir da leitura de bibliografia sobre o Naturalismo (Guinsburg \& Faria, 2017) e a história do Naturalismo em Portugal (França, 2002), foi possível compreender a forma como esta corrente artística surgiu e influenciou os artistas portugueses

\footnotetext{
${ }^{1}$ No acervo do Museu Nacional Grão Vasco existe uma pintura de Júlio Ramos, denominada Paisagem, e no Museu Nacional de Arte Antiga, estão quatro exemplares, denominados Tempestade próxima, Manhã, Azenha do Bicho, no Ave e Sinfonia de cor. O Museu Nacional de Soares dos Reis possui igualmente obras deste autor, embora não tenha sido conseguida a informação sobre quais as existentes
} 
A última fase deste estudo procurou compreender as motivações que levaram Maria da Soledade Malvar a adquirir a obra "Um riacho" (Milheiroz). Para responder a esta questão, tornou-se importante aprofundar os conhecimentos sobre Soledade Malvar e os seus hábitos de colecionar. Para este fim, foram consultados vários documentos existentes no acervo documental da Casa-Museu, tendo sido utilizada, sobretudo, a brochura intitulada Casa-Museu Soledade Malvar/Roteiro (Rede de Museus de Vila Nova de Famalicão, 2002), visto que este documento menciona a grande parte da informação referida nos restantes. A informação recolhida mostrou-se um pouco escassa, o que implicou a necessidade de utilizar outros métodos. É neste sentido que surge a visita à Quinta dos Cónegos, no concelho da Maia, local onde a colecionadora anteriormente residiu, e que permitiu recolher pequenos dados sobre esta fase da vida de Soledade Malvar.

Finalmente, de forma a complementar os dados biográficos sobre Maria da Soledade, realizaram-se duas entrevistas. O objetivo inicial era entrevistar um número maior de pessoas que tivessem criado diferentes tipos de relacionamento com a colecionadora. Apesar destes intentos, a propagação do coronavírus SARS-CoV-2 impossibilitou a concretização de um vasto número de entrevistas, em tempo útil.

\section{Resultados e discussão}

De um modo geral, os dados recolhidos permitiram desenvolver as referidas quatro fases de estudo da pintura. Porém, algumas questões relacionadas com a história deste objeto não puderam ser respondidas. Os esforços direcionados para a procura dos locais onde Soledade Malvar adquiria as suas pinturas não resultaram em quaisquer informações. Isto deve-se à falta de resposta, por parte dos antiquários e leilões contactados e à inexistência de qualquer documentação que pudesse comprovar a compra dos objetos. 
No que diz respeito à história da CMSM e das suas transformações, sobretudo aquelas executadas durante o período de obras entre 2000 e 2002, a recolha de referências foi pouco determinante para a investigação, visto que as únicas dissertações que a referem (Moreira, 2006; Ponte, 2007) descrevem-na apenas de forma breve. Pelo contrário, a consulta do acervo documental da CMSM revelou-se fundamental. Inclui vários documentos que referem as alterações realizadas durante as obras de $2000 \mathrm{e}$ 2002 e o processo do armazenamento e transporte dos objetos durante esta fase. Os dois conjuntos consultados de fotografias antigas, de datações distintas, que retratam a CMSM numa fase anterior às obras referidas, revelaram-se importantes para o estudo do edifício, permitindo conhecer o aspeto anterior do edifício e estabelecer uma comparação com o atual. Ao contrário do que se esperava, este processo de comparação tornou-se difícil, uma vez que, para além da disposição dos objetos ter sido alterada significativamente, muitas das fotografias demonstram apenas uma pequena parte de cada divisão do edifício. Ora, esta situação resultou na dificuldade em identificar as divisões da casa e obrigou, em último recurso, a comparar apenas os espaços que foram reconhecidos, que correspondem aos atuais espaços expositivos da CMSM. Em contexto das entrevistas, foi possível compreender as motivações que levaram à alteração significativa da organização e disposição das divisões do edifício. Conclui-se que esta alteração foi efetuada num momento em que várias peças, anteriormente doadas, se encontravam na posse de Maria da Soledade e por sua vontade. As modificações devem-se, igualmente, ao facto desta disposição dos objetos ter sido realizada de uma forma intuitiva, não seguindo, concretamente, a apresentação que o edifício anteriormente mostrava.

Parte do Relatório de Estágio desenvolvido focou-se, também, na descrição e reflexão crítica sobre as atividades complementares de estágio, realizadas, maioritariamente, na CMSM. Estas foram seccionadas em 4 tipologias distintas, denominadas "encontros científicos", "estudo de coleções", "serviços educativos" e "tratamento arquivístico". Entre estas, incluem-se as atividades da RMVNF, que permitiram conhecer a dinâmica entre os museus que a integram. Aliada a esta atividade, também a visita aos vários 
museus pertencentes a esta Rede, trouxe a oportunidade de conhecer o património famalicense e a forma como estes se envolvem com a comunidade de Vila Nova de Famalicão. Uma grande parte das atividades desenvolvidas foi executada com o intuito de recolher informação útil para a investigação.

A última fase de investigação foi desenvolvida juntamente com a colega de estágio, Thalita Sbragio, e diz respeito à leitura e revisão das fichas de inventário da CMSM, o que permitiu conhecer o tipo de material de suporte que os objetos de pintura apresentam a sua localização dentro do museu. A tarefa permitiu, ainda, identificar lacunas e surgiu a necessidade de inserir informação. Para este exercício foram consultadas as normas de gestão e inventariação de coleções SPECTRUM (Collections Trust, 2014) e do IPM - Instituto Português dos Museus (Instituto Português dos Museus, 2000-2010), para além do manual de apoio ao preenchimento de dados do sistema In Patrimonium. Net. Foram adotadas as normas estabelecidas por este sistema, já utilizadas pela RMVNF nas suas instituições museológicas. Desta atividade resultou um conjunto de novas fichas e um manual de auxílio ao preenchimento deste tipo de documentos. A este manual foi acrescentado um glossário, com a definição de alguns termos relacionados com as técnicas de pintura.

\section{Considerações finais}

Apesar da existência de uma quantidade significativa de instituições museológicas em Portugal, grande parte das mesmas não possui capacidades económicas para apostar no estudo dos objetos que possuem. Este é o caso da CMSM que, até então, não tinha desenvolvida nenhuma investigação aprofundada sobre os objetos da sua coleção. Tal situação reflete-se ao nível da documentação sobre as coleções. Tendo em conta este contexto, a realização do estágio referido e a redação do respetivo relatório permitiram colmatar algumas destas lacunas. Assim, a investigação desenvolvida torna-se um válido contributo para a instituição, globalmente. 


\section{Agradecimentos}

A autora expressa os seus agradecimentos às suas orientadoras e aos vários membros da Rede de Museus de Vila Nova de Famalicão, pelo acolhimento e pelo apoio na elaboração do estágio. Um especial agradecimento à colega Thalita Sbragio, pelo trabalho de parceria na execução das atividades e na realização do processo de leitura e revisão das fichas de inventário da CMSM

\section{Referências}

- Appadurai, A. (1986). Introduction: commodities and the politics of value. In A. Appadurai (Ed.), The Social Life of Things (pp. 3-60). Cambridge: Cambridge University Press.

- Batchelor, R. (2003). Not looking at kettles. In S. Pearce (Ed.), Interpreting Objects and Collections (pp. 139-143). London and New York: Routledge.

- Câmara Municipal de Vila Nova de Famalicão (s.d.). Acta. Câmara Municipal. V. N. Famalicão.

- Câmara Municipal de Vila Nova de Famalicão (1998). Escritura de Doação. Câmara Municipal. V. N. Famalicão.

- Câmara Municipal de Vila Nova de Famalicão (2000). Concurso Público "Remodelação de Edifício a Casa-Museu Soledade Malvar". Câmara Municipal. V. N. Famalicão.

- Câmara Municipal de Vila Nova de Famalicão (2002). Cultura e Turismo: O Perfil de Maria da Soledade Malvar [Press release]. Disponível em: https://www.cmvnfamalicao.pt/_o_perfil_de_maria_da_soledade_malvar 
- Câmara Municipal de Vila Nova de Famalicão (2012). Cultura e Turismo: Museus de Famalicão Unem-se em Rede e Apostam no Turismo. [Press release]. Disponível em: https://www.cmvnfamalicao.pt/_museus_de_famalicao_unemse_em_rede_e_apostam_no_tur ismo

- Collections Trust (2014). Spectrum 4.0 - Padrão para Gestão de Coleções de Museus do Reino Unido (Collections Trust). São Paulo: Secretaria de Estado da Cultura; Associação de Amigos do Museu do Café; Pinacoteca do Estado de São Paulo.

- Elliot, R. (2003). Towards a material history methodology. In S. Pearce (Ed.), Interpreting Objects and Collections (pp. 109-124). London and New York: Routledge.

- França, J. (2002). A Arte Portuguesa de Oitocentos. Lisboa: Instituto de Cultura e Língua Portuguesa.

- Guinsburg, J., \& Faria, J. (2017). O Naturalismo. S. Paulo, Brasil: Editora Perspectiva S.A.

- Günhan, A. (2011). From Houses to House Museums: Architectural Representation of Different Narrations. (Dissertação de Mestrado. The Graduate School of Natural and Applied Sciences of Middle East Technical University, Turkey). Disponível em: https://open.metu.edu.tr/handle/11511/20883

- Instituto Português dos Museus (2000-2010). Normas de Inventário. Disponível em: http://www.matriznet.dgpc.pt/matriznet/Normas/nventario.aspx.

- Kopytoff, I. (1986). The cultural biography of things: commoditization as process. In A. Appadurai (Ed.), The Social Life of Things (pp. 64-91). Cambridge: Cambridge University Press. 
- Leiloeira S. Domingos (s.d.). LEILÕES / Leilão 2019 - 93: Júlio Ramos. Disponível em:

https://www.leiloeirasaodomingos.pt/leilao/2019.93/0/1/2/05/J\%C3\%BAlio\%2 ORamos $/ 0 / 0$

- Moreira, M. R. (2006). Da Casa ao Museu: Adaptações Arquitectónicas nas Casas-museu em Portugal. (Dissertação de Mestrado, Universidade do Porto, Portugal). Disponível em:

https://sigarra.up.pt/faup/pt/pub_geral.pub_view?pi_pub_base_id=274165

- Navalho, S. S. (2020). Casa-Museu Soledade Malvar. Edifício, Coleção e Inventário. (Dissertação de Mestrado, Universidade do Porto Portugal). Disponível em: https://hdl.handle.net/10216/130664

- Pamplona, F. (1991). Dicionário de Pintores e Escultores Portugueses ou que Trabalham em Portugal. Porto: Livraria Civilização Editora.

- Pearce, S. M. (2003a). Collecting reconsidered. In S. M. Pearce (Ed.), Interpreting Objects and Collections (pp. 193-204). London and New York: Routledge.

- Pearce, S. M. (2003b). Objects as meaning; or narrating the past. In S. M. Pearce (Ed.), Interpreting Objects and Collections (pp. 19-29). London and New York: Routledge.

- Pearce, S. M. (2003c). Thinking about things. In S. M. Pearce (Ed.), Interpreting Objects and Collections (pp. 125-132). London and New York: Routledge.

- Ponte, A. (2007). Casas-museu em Portugal: Teorias e Práticas. (Dissertação de Mestrado, Universidade do Porto, Portugal). Disponível em: https://www.academia.edu/42249970/PONTE_2007__Disserta\%C3\%A7\%C3\%A3o_de_Mestrado

- Ponte, A. (2019). Casas-museu. Entre o conceito e o modelo de ação. Da constituição ao modelo de investigação. In M. J. Monge, Coleção Património a Norte 1. 10 Anos de Reflexão sobre Casas-Museu em Portugal (pp. 19-34). 
Porto: Direção Regional de Cultura do Norte - Ministério da Cultura, Comité Internacional do ICOM - Portugal e DEMIST.

- Prown, J. D. (2003). Mind in matter. An introduction to material culture theory and method. In S. Pearce (Ed.), Interpreting Objects and Collections (pp. 133138). London and New York: Routledge.

- Rede de Museus de Vila Nova de Famalicão (2002). Casa-Museu Soledade Malvar/Roteiro [Brochura]. Vila Nova de Famalicão: Câmara Municipal de Vila Nova de Famalicão.

- Rede de Museus de Vila Nova de Famalicão (2018). Rede de Museus Vila Nova de Famalicão. A Nossa Identidade. O Nosso Futuro [Brochura]. Vila Nova de Famalicão: Câmara Municipal de Vila Nova de Famalicão.

- Rede de Museus de Vila Nova de Famalicão (2019). Definir a missão... da necessidade ao desafio. Ser e Fazer Museu no Século XXI, 1, 49-51.

- Salão Silva Porto (1943). Exposição Retrospectiva e de Homenagem ao Mestrepintor Júlio Ramos. Porto: s.n.

- Universidade Digital/Gestão de Informação (2009). Antigos Estudantes Ilustres da Universidade do Porto - Júlio Ramos. Disponível em: https://sigarra.up.pt/up/pt/web_base.gera_pagina?p_pagina=antigos\%20estud antes\%20ilustres\%20-\%20j\%c3\%balio\%20ramos 